Anuario Latinoamericano Ciencias Políticas

y Relaciones Internacionales

vol. 8, 2019

pp. 101-114

\section{Mujeres indígenas y representación política: la dimensión simbólica de su presencia en el espacio público}

\author{
Indigenous Women and Political Representation. \\ The Symbolic Dimension of Their Presence \\ in the Public Space
}

\author{
Andrea Ivanna Gigena * \\ FACULTAD DE CIENCIA POLÍTICA Y RELACIONES INTERNACIONALES \\ UNIVERSIDAD CATÓLICA DE CÓRDOBA, ARGENTINA \\ $\triangle$ aigigena@conicet.gov.ar \\ https://orcid.org/0000-0002-0612-3146
}

\section{RESUMEN}

El presente artículo aborda la representación política de las mujeres indígenas a partir de considerar su dimensión simbólica. Se parte del supuesto de que los análisis sobre la representación descriptiva y sustantiva de grupos subrepresentados en el Estado (como indígenas y mujeres) son importantes, pero insuficientes para comprender el modo en que se desarrolla la politización y la participación de las mujeres indígenas en el espacio público. Se analiza, entonces, cómo se configura la representación simbólica y los usos que ésta asume entre los pueblos indígenas (como afirmación o como excusa) y en el Estado (como folklorización). El análisis está basado en información secundaria (documental) para la región latinoamericana e información primaria: entrevistas en profundidad e historias de vida realizadas, entre los años 2001 y 2017, a referentes sociales y políticos/políticas de diferentes pueblos indígenas en Argentina, Bolivia, Chile y Panamá.

PALABRAS CLAVE: mujeres indígenas, representación política, representación simbólica.

\section{ABSTRACT}

This article discusses the political representation of indigenous women considering its symbolic dimension. It assumes that analyses about the descriptive and substantive

* Doctora en Ciencias Sociales (UBA), Magister en Administración Pública (UNC), Licenciada en Ciencia Política (UCC). Investigadora CONICET. Investigadora Visitante en el Centro de Estudios Interculturales e Indígena de Chile y en la Facultad de Humanidades de la Universidad de Panamá. Profesora de grado y posgrado en el área de Metodología de la Investigación. 
Dossier América Latina: género y política representation of groups underrepresented in the State (such as indigenous people and women) are important but insufficient to understand the way in which the politicization and participation of indigenous women in the public space develops. Hence, what is analyzed is how symbolic representation is shaped and the uses it acquires among indigenous peoples (as assertion or excuse) and within the State (as folklorization). This analysis is based on secondary (documentary) information for the Latin American region and primary information: life stories and in-depth interviews, carried out between the years 2001 and 2017, with social and political figures of different indigenous peoples from Argentina, Bolivia, Chile and Panama.

KEYWORDS: indigenous women, political representation, symbolic representation.

\section{Introducción}

La representación política de las mujeres ha estado circunscripta, predominantemente, al análisis de la "representación descriptiva" y la "representación sustantiva" en los poderes del Estado (sobre todo el legislativo). En similar sentido se han orientado los esfuerzos de incidencia y acción política de los organismos internacionales y los hacedores de políticas públicas a nivel nacional.

Los conceptos de representación sustantiva y descriptiva provienen, por cierto, del estudio que Hanna Pitkin publicó en 1967 (traducido al español en 1985), apropiado con pocos matices en la Ciencia Política. Con el primer término, se hace referencia a la "correspondencia en la representación de la ciudadanía y los órganos representativos" (Freidenberg y Alva Huitrón, 2017, p. 4). Es decir, se observa en qué medida los sistemas de representación (multinivel) reflejan la composición demográfica, social, cultural, económica, etc. de una sociedad. Con el segundo, se remite a la “... representación de los intereses del electorado desde los actores de la representación" (Freidenberg, Alva Huitrón 2017, p. 4). El foco analítico está puesto en la relación (y su correspondencia) entre representante y representados-as.

Ahora bien, la reducción de la representación femenina a estas "perspectivas" (Pitkin, 1985) descriptivas y sustantivas, presenta algunos problemas cuando consideramos a las mujeres indígenas. Por un lado, y de modo casi excluyente, subyace el supuesto de la homogeneidad del colectivo femenino afectado por las brechas de género. Así, las mujeres apenas aparecen diferenciadas por variables poblacionales (edad, nivel educativo, estado civil, lugar de procedencia, etc.) o por variables de trayectorias políticas-ideológicas o políticas-partidarias. Por otro lado, la "representación" queda reducida a un Estado-nación monocultural. Con esto se desconoce la heterogeneidad constitutiva de nuestras sociedades latinoamericanas, la coexistencia de grupos de diversas procedencias históricas - prehispánico, colonial y republicano- que no han sedimentado en una matriz política, social y/o cultural común, pero 
que participan (o quieren participar) de la estructura de poder, autoridad y representación del Estado (de modo integrado, complementario o en tensión).

Todo esto, puesto en diálogo con lo expresado por muchos-as referentes de los pueblos indígenas, nos invita a revisitar una de las perspectivas menos abordadas del estudio de Hanna Pitkin, el de la representación simbólica, justamente para: "hacer justicia a las diversas aplicaciones más destacadas que se han hecho de la representación en contextos diferentes" (Pitkin, 1985, p. 11).

Comenzaré primero por presentar los rasgos más sobresalientes (aunque no exhaustivos) de la representación descriptiva y sustantiva con relación a las mujeres y los pueblos indígenas. Esto nos permitirá, luego, un mejor acercamiento al abordaje de la "representación simbólica" de las mujeres-indígenas en el espacio público: su configuración y "usos".

\section{Las mujeres indígenas en los estudios y acciones sobre la representación política femenina}

Sobre la representación descriptiva se ha desarrollado un corpus de estudios especializados importante que transita desde el análisis de los "obstáculos" y "condicionantes" para el acceso de las mujeres a un cuerpo legislativo o algún órgano ejecutivo multinivel hasta los estudios sobre las medidas de acción afirmativa o discriminación positiva que promueven o garantizan una mejor representación (tipos, contextos y modos de emergencia, diseños, efectividad, argumentos a favor y en contra para su implementación, etc.).

Siguiendo a Archenti (2002), las medidas de acción afirmativa pueden ser de tres tipos: a) programas de igualdad de oportunidades; b) espacios de la mujer en el ámbito ejecutivo y c) leyes de cuotas o de paridad para procesos eleccionarios a cargos de representación popular. Asimismo, en relación con este último, los mecanismos que suelen implementarse son: escaños reservados, leyes electorales de cuota y cuotas de partidos políticos (Krook, 2008).

Actualmente, como resultado de un proceso que ganó su mayor impulso en la década de los 90, en toda Latinoamérica y el Caribe hispano se registran acciones en los tres tipos mencionados por Archenti (2002).

En primer lugar, todos los países cuentan con alguna política y acciones orientadas a promover la igualdad de género; en algunos países sancionadas como leyes, en otros como programas o planes (y en algunos se registran ambos instrumentos).

En segundo lugar, todos los países tienen organismos especiales de la mujer en el ámbito del poder ejecutivo, los denominados: "Mecanismos Nacionales de la(s) Mujer(es)" o "Mecanismos para el Adelanto de la Mujer". La única excepción es la Federación de Mujeres Cubanas, que es una organización no gubernamental, que fue reconocida, sin embargo, como Mecanismo Nacional por el Estado cubano. En correspondencia, los organismos internacionales hacen los mismo.
Mujeres indígenas y representación política: la dimensión simbólica de su presencia en el espacio público

Andrea Ivanna Gigena 
Dossier América Latina: género y política
Los Mecanismos Nacionales son los responsables, a nivel internacional, de cumplir con los mandatos emanados de la Plataforma de Acción de Beijing; de la Comisión Interamericana de Mujeres de los Estados Americanos (CIMOEA), de la Convención de Belém do Pará, y de otros mecanismos regionales específicos. A nivel interno deben diseñar y ejecutar la política nacional para mujeres, promoviendo la igualdad de oportunidades entre mujeres y varones en el Estado y la sociedad en general. Se espera, asimismo, que sean promotores y garantes de los derechos de las mujeres y trabajan por su ampliación.

En tercer lugar, salvo Cuba y Guatemala, todos los países de Latinoamérica y el Caribe hispano han dictado alguna medida para promover una mayor participación de mujeres en procesos eleccionarios para cargos de representación popular (leyes de cuotas o paridad).

Ahora bien, en todas estas acciones, la incorporación del tema indígena o un enfoque étnico ha sido una circunstancia excepcional. En relación con los programas de igualdad de oportunidades, según datos de la CEPAL (2013) sólo 10 países registran acciones que incluyen la dimensión étnica.

En términos generales, los asuntos relativos a las mujeres indígenas se abordan desde tres perspectivas diferentes: como tema central del plan, asumiendo que a la inequidad de género se agrega la étnica, y encarándolas mediante acciones conjuntas, como en el Estado Plurinacional de Bolivia, el Ecuador, Guatemala, Honduras y el Paraguay. Una segunda perspectiva consiste en considerar a las mujeres indígenas un grupo prioritario e incluir objetivos y lineamientos específicos, tal como en los planes de Chile, El Salvador, Panamá y el Perú, entre otros. Finalmente, un tercer enfoque es el de México, en el que ambos tipos de discriminación (étnica y de género) se abordan mediante planes especificos paralelos subsumidos en el Plan Nacional de Desarrollo. (CEPAL 2013, pp. 123-124) [el resaltado es propio]

Con respecto a los espacios de la mujer en el poder ejecutivo, en algunos Estados se han constituido instancias de coordinación intersectorial con los órganos a cargo del tema indígena; en otros se han creado oficinas o dependencias de bajo rango, o programas ad hoc en los Mecanismos Nacionales de la Mujer (Chile, Costa Rica, Ecuador, Venezuela).

Finalmente, en ninguna de las leyes de cuotas o de paridad se considera a las mujeres indígenas de modo particular. De modo llamativo, el caso boliviano que suele ser presentado como pionero en materia de paridad política (tanto en términos formales como descriptivos), nunca es analizado a partir de considerar lo "étnico" como uno de los factores explicativos de la mentada paridad. Esta dimensión aparece, a veces, para explicar la brecha en la representación sustantiva entre las mujeres no indígenas y las mujeres indígenas.

En relación con la representación sustantiva se ha estudiado, por ejemplo, la conformación de las agendas legislativas en temas, necesidades y derechos de interés para las mujeres, así como también el proceso de presentación de las 
iniciativas y las estrategias orientadas a su sanción en los parlamentos. Sobre estos puntos, indican Rodríguez Gustá y Madera:

Un aporte significativo de las indagaciones politológicas es haber identificado que los procesos de representación sustantiva son complejos (porque el vínculo entre ideología partidaria y perspectiva de género no es unívoco), contingentes (dependen de coyunturas politicas que hacen que los temas de género sean "moneda de cambio") y contradictorios (los "intereses" de las mujeres son materia de disputa). (2016, p. 358)

Asimismo, estas autoras estudiaron, desde un enfoque sociológico y para Latinoamérica y el Caribe, cómo esas agendas legislativas se constituyeron por fuera de la dinámica legislativa propiamente dicha, vinculando legisladoras con diferentes organizaciones de la sociedad (nacional e internacional).

La escasa presencia de mujeres indígenas (como veremos en breve) en los cargos de representación popular vuelve difícil considerar la representación sustantiva. En todo caso, sabemos por qué está documentado bibliográficamente, particularmente para los últimos años, que en general las mujeres indígenas mantienen una vinculación distante con las mujeres y las feministas de la sociedad mayoritaria: porque no representan sus derechos específicos, porque no consideran los derechos colectivos de los pueblos y porque se erigen en "representantes" de las mujeres indígenas sin darles "voz".

\section{Las mujeres indígenas en los estudios y acciones sobre la representación política indígena}

Los estudios sobre la representación descriptiva y sustantiva en clave étnica son significativamente menores. Por un lado, el tema indígena ha estado prácticamente ausente en los análisis teóricos y empíricos de la Ciencia Política (Gigena, 2017), disciplina particularmente enfocada en el estudio de la representación política. Por otro lado, históricamente la presencia indígena en los órganos del Estado en la región no ha sido significativa. Solo recientemente encontramos algunos casos paradigmáticos.

Contamos, sin embargo, con alguna información recopilada y producida por observatorios y estudios de las propias organizaciones indígenas internacionales u organismos internacionales. Según datos de la CEPAL (2014), entre el 2010 y el 2015 hubo participación de indígenas en órganos legislativos solo en 6 países: Bolivia, Ecuador, Guatemala, México, Nicaragua y Perú. El número total de parlamentarios-as en estos países ascendía a 93 indígenas (71 varones y 22 mujeres). En ningún caso esto número reflejaba la proporción real de población indígena en sus respectivas sociedades. Asimismo, salvo en Bolivia y Guatemala, donde la representación indígena alcanzó el 31,5 \% y el 12 \% del total de parlamentarios-as (respectivamente), en el resto de los países nunca
Mujeres indígenas y representación política: la dimensión simbólica de su presencia en el espacio público

Andrea Ivanna Gigena 
Dossier América Latina: género y política superó el 7 \%. Y el máximo porcentaje de mujeres-indígenas registrado en un parlamento fue del 6,9\% en Bolivia.

Esas mismas organizaciones y organismos internacionales que recopilan estos datos nos brindan diagnósticos, muy generales y exploratorios, sobre "dificultades" "barreras" y "desafíos" que atraviesan los indígenas para alcanzar algún nivel de representación política y, con ello, ejercer la defensa de sus derechos específicos desde las estructuras estatales.

Si comparamos los tipos de medidas de acción afirmativa que Archenti (2002) identifica para las mujeres con las establecidas para pueblos indígenas, genéricamente coinciden: a) programas de igualdad de oportunidades (y no discriminación racial), b) espacios de asunto indígenas en el ámbito ejecutivo, y c) mecanismos que favorecen el acceso a cargos de representación popular mediante elecciones.

En primer lugar, hasta el 2013 en 6 Estados se habían dictado leyes nacionales contra el racismo y la discriminación (CEPAL, 2013) que, genéricamente, siempre incluyen a las poblaciones indígenas. Sólo en Ecuador esa ley estaba específicamente referida a la población indígena.

En segundo lugar, 16 países de Latinoamérica y el Caribe hispano tienen en el poder ejecutivo organismos especiales dedicados a los asuntos indígenas, la mayoría de ellos creados en la década de los 2000. Algunos países, además, tienen cierta "dispersión" de mecanismos (Bolivia, Colombia, Ecuador, Guatemala y Panamá); a diferencia de lo que ocurre con los Mecanismos Nacionales de las Mujeres que son un órgano único a nivel nacional (aunque sus dependencias pueden estar descentralizadas en el territorio nacional).

En tercer lugar, algunos países de Latinoamérica y el Caribe hispano registran la sanción de medidas para promover la mayor participación de indígenas en procesos eleccionarios para cargos de representación popular. Los tipos de mecanismos previstos por países son:

a) Escaños (o asientos) reservados: Bolivia, Colombia y Venezuela (CEPAL, 2013; PNUD, 2016)

b) Cuotas electorales: Perú ha reglamentado las “cuotas nativas" para elecciones regionales y municipales (que se combinan, además, con las cuotas de género establecidas a nivel nacional)

c) Mapas electorales o redistritación (PNUD, 2016) o distritos étnicos: México. Y aquí deben considerarse, también, los casos de las Comarcas Indígenas en Panamá y las Regiones Autónomas en Nicaragua, que si bien fueron creadas para otros fines (delimitación territorial y reconocimiento de autogobierno), han tenido efecto sobre la representación indígena en el parlamento nacional en la medida que los distritos electorales coinciden con los límites comarcales y autonómicos. En caso nicaragüense, además, se combina la representación indígena con cupos para mujeres.

d) En algunos países se han creado, autónomamente, partidos políticos indígenas (Ecuador, Nicaragua y Guatemala. En Chile "Wallmapuwen"). Estos partidos logran incorporar en sus plataformas la dimensión colectiva de 
los derechos y las demandas indígenas, pero casi nunca logran obtener representación parlamentaria a nivel nacional. A veces logran mejores resultados a nivel local.

La incorporación o la transversalización de la dimensión (o enfoque) de género en las acciones mencionadas en este apartado está prácticamente ausente.

En los programas de igualdad de oportunidades o no discriminación racial, las acciones que articulaban ambas dimensiones (etnia y género) fueron referenciadas en el apartado anterior.

Para los espacios de asuntos indígenas creados en el ámbito del poder ejecutivo, se mencionan un conjunto de medidas sectoriales (que no se pueden considerar exhaustivas) orientadas a apoyar o fortalecer a grupos de mujeres de comunidades o pueblos indígenas determinados y en temáticas puntuales como: tareas productivas, organizativas, educativas, y de salud sexual y reproductiva (CEPAL, 2013; Gigena, de Cea, 2017).

Finalmente, y en relación con los mecanismos que favorecen el acceso a cargos de representación popular mediante elecciones, salvo en los casos ya referidos de Perú y de Nicaragua, en ninguna de las otras acciones mencionadas, se ha considerado de modo particular a las mujeres indígenas. Justamente, considerando la representación política parlamentaria, Cunningham y Sena afirman:

El análisis de las diversas estrategias promovidas tanto por mujeres en general como por pueblos indígenas para aumentar su respectiva presencia en los parlamentos permite concluir que estas no han facilitado la participación de mujeres indígenas. En el caso de los "asientos asignados", estos han sido ocupados por hombres y en el caso de las cuotas o circunscripciones especiales tampoco han beneficiado a las mujeres indígenas. (Cunningham, Sena, 2013, p. 11)

\section{Pensar la representación de las mujeres indígenas: más allá y más acá del Estado}

Según Pitkin (1985), la representación simbólica establece un vínculo entre los-as representantes y los-as representados-as que no exige semejanza o reflejo entre uno y otro (como sí la representación descriptiva). Es una relación diferente que supone, más bien, la creación de un símbolo.

Para explicar mejor por qué creo pertinente recuperar esta noción, comenzaré por presentar brevemente los diferentes ámbitos de participación que se reconocen para los pueblos indígenas, desde diversos enfoques.

Una sistematización académica y politológica (Fuentes, Sánchez, 2018) considera que los tipos posibles de participación política surgen de una combinación de niveles: nacional, regional, o municipal-local y ámbitos: autonomías indígenas, instituciones representativas o poder ejecutivo. Los espacios
Mujeres indígenas y representación política: la dimensión simbólica de su presencia en el espacio público

Andrea Ivanna Gigena 
Dossier América Latina: género y política institucionales de representación constituidos en estos entrecruzamientos no son excluyentes y hasta pueden ser complementarios. Los autores quieren enfatizar que las organizaciones autónomas indígenas no necesariamente se oponen a los espacios de representación de los Estado-nación republicanos.

Otra sistematización sobre los ámbitos de participación política de los indígenas la ofrecen los organismos internacionales que reconocen, de modo simplificado, cuatro niveles de incumbencia.

El nivel local y comunitario: interno, territorial, donde pueden concurrir las estructuras comunitarias propias, ancestrales y otras organizaciones orientadas a mejorar los servicios públicos, desarrollar actividades culturales, productivas, comerciales, etc.

El nivel municipal: forma de gobierno local del Estado republicano que, con la transición a la democracia primero y los procesos de descentralización después, se vieron fortalecidos como ámbitos de resolución de las demandas inmediatas. En algunos de ellos, la densidad de población indígena ha generado la creación de oficinas y programas especiales. Asimismo, se trata del espacio donde los partidos políticos indígenas han tenido mejores resultados y donde se buscan actualizar, incluso sobre las estructuras políticas estatales, algunos mecanismos de gobierno de la tradición indígena.

El nivel nacional: remite tanto los diferentes ámbitos de gobierno del Estado republicano (ejecutivo, legislativo y judicial) como a la articulación de organizaciones indígenas locales, regionales o por pueblos.

El nivel internacional: aquí se destaca: a) el involucramiento de indígenas en instancias de preparación, participación y seguimiento de conferencias, convenciones y tratados internacionales; b) la participación como observadores-as y/o asesores-as en diversos organismos internacionales (particularmente del sistema de las Nacionales Unidas); la creación del Fondo para el Desarrollo de los Pueblos Indígenas de América Latina y El Caribe (FILAC), en tanto instancia de participación paritaria con los Estados de la región (Cunningham, Sena, 2013; CEPAL, 2014; PNUD, 2016).

A su vez, Eileen Mairena (2002) distingue los siguientes cuatro niveles. El comunitario, de carácter territorial, que incluye a la familia, las organizaciones indígenas mixtas y/o las organizaciones indígenas de mujeres. El local, que puede incluir: los espacios tradicionales, los gobiernos locales o municipales o los gobiernos autonómicos. El nacional, que incluye tanto la representación política en el Estado (a nivel legislativo o en los organismos de asuntos indígenas que integran el poder ejecutivo) y las organizaciones indígenas nacionales (que en general articulan varios pueblos). Finalmente, el internacional que considera la participación en redes de articulación indígena (Campaña Continental de los 500 años y el Consejo Mundial de los Pueblos Indígenas); en el Grupo de Trabajo sobre Poblaciones Indígenas de la ONU y; en otras organizaciones internacionales no gubernamentales como: el Consejo Mundial de Pueblos Indígenas (CMPI), el Fondo para el Desarrollo de los Pueblos Indígenas de América Latina y El Caribe (FILAC). 
Como específicamente vinculados a mujeres indígenas Mairena menciona el Foro Internacional de Mujeres Indígenas (FIMI) y el Enlace Continental de Mujeres Indígenas de las Américas (ECMIA). Podríamos agregar, además, la Alianza de Mujeres Indígenas de Centroamérica y México y la Red de Mujeres Indígenas sobre Biodiversidad de América Latina y el Caribe (RMIB-LAC).

Me interesa esta clasificación de Mairena por dos razones. Por una parte, ella es una activista indígena y su trabajo refleja la visión consolidada y consensuada entre los-as dirigentes históricos-as indígenas de Latinoamérica y el Caribe hispano sobre los ámbitos de actuación de los pueblos indígenas. Pero, por otra parte, a diferencia de las clasificaciones anteriores, Mairena considera los niveles más reductivos íntimos (el nivel molecular) donde se origina la representación política simbólica de las mujeres indígenas: el ámbito comunitario.

\section{La representación simbólica: afirmación, excusa o folklore}

Es muy frecuente, tanto entre varones como entre mujeres de diferentes pueblos indígenas que ante la pregunta por las razones y/o causas que dificultan el acceso de las mujeres indígenas a cargo de representación popular, las respuestas giren alrededor de la idea de que las indígenas ejercen una representación política amplia, pero en el nivel comunitario. Por un lado, en las familias, en su condición de agentes "naturales" de la reproducción cultural (y biológica) de sus pueblos. Por otro lado, ejerciendo el rol complementario en la autoridad indígena dual, según el sistema de prácticas ancestrales. Finalmente, presidiendo las organizaciones indígenas territoriales, mixtas y/o de mujeres.

Esto no lleva a los planteos iniciales del artículo. Si reducimos la representación política al ejercicio de funciones emanadas de elecciones para cargos en las instituciones representativas del Estado $-\mathrm{y}$ la abordamos a partir de la dimensión descriptiva o sustantiva- se cancela la posibilidad de pensar lo comunitario, al que tan acertadamente se refiere Mairena (2002), como un espacio de ejercicio de la representación femenina.

Se pierde, asimismo, la posibilidad de entender que, en sociedades heterogéneas y en el marco del reconocimiento de los derechos que, por ejemplo, establece el Convenio 169 de la OIT, los caminos de la representación no pueden circunscribirse a un modo único de elección. De hecho, uno de los puntos más controvertidos a la hora de resolver los procedimientos para hacer efectivas las medidas que favorecen una mayor participación de indígenas en cargos de representación popular es el "sistema electoral": si se respetará (y de qué manera) los modos tradicionales de elección de candidaturas y representantes.

Ahora bien, estas respuestas inmediatas dadas por los-las indígenas permiten considerar más detalladamente el modo en que se configura la representación simbólica.
Mujeres indígenas y representación política: la dimensión simbólica de su presencia en el espacio público

Andrea Ivanna Gigena 
Dossier América Latina: género y política
La erosión del sistema de vida y autoridad tradicional indígena a lo largo de los siglos ha generado, en las últimas décadas, una vuelta a la cultura (su "preservación", su "recuperación”). Asimismo, la expansión del despojo que conlleva la modernidad capitalista globalizante ha generado una acción defensiva de los territorios, los recursos naturales y los modos de vida indígena. En todos estos procesos las mujeres indígenas tienen un protagonismo indiscutible. Son garantes "materiales" e "inmediatas" de la "defensa de la vida" y la reproducción de sus comunidades.

Esto, privilegiadamente, las instituye como "representantes" simbólicas de la supervivencia de sus Pueblos, ante los suyos y ante la sociedad mayoritaria en la que están insertos. Es por esto por lo que la reivindicación del ámbito comunitario, de los espacios más íntimos de la vida social (la "familia”), cómo instancias de representación política es un acto de afirmación (de politización) de la identidad.

Esta afirmación no está exenta de problemas, claro. Las estrategias que las mujeres indígenas han desarrollado para "mejorar sus posibilidades de participación comunitarias" (CEPAL, 2014) demuestran tanto las dificultades como la politicidad de la representación que construyen y encarnan.

Entonces medianamente esas mujeres también están hablando, están teniendo su voz al interior de la comunidad, el problema de que como todo lo que sucede en la comunidad, no es conocido a nivel regional, no es conocido a nivel nacional y menos a nivel internacional. (Ciudad de Temuco, Chile, junio de 2016, entrevista realizada por la autora)

Otras veces, entre varones y mujeres indígenas, pero particularmente entre los primeros, esta afirmación transmuta en una excusa, para no promover una mayor participación política de las mujeres indígenas (en cualquier tipo de representación).

Por ejemplo, entre indígenas varones es recurrentes la referencia a que, si bien la representación femenina en los espacios extracomunitarios es muy baja, es significativa (y "mayoritaria") al interior de las organizaciones comunitarias. Se trata, muchas veces, de interpretación que no está acompañada de evidencia o, incluso, que la contradice (Gigena, 2018).

Algo similar ocurre con la mención a la "complementariedad". Muchas veces es utilizada de modo retórico para demostrar la importancia de la representación femenina, al mismo tiempo que se reafirma con ella el statu quo de las relaciones de género al interior de los pueblos. La "complementariedad" entre las mujeres indígenas, en tanto, suele ser una de las estrategias a las que apelan para alcanzar mayor participación y/o representación comunitaria.

Lo que no valoran estas posiciones es la importancia y la potencia de la representación simbólica en el ámbito comunitario, pero también si logran trascenderlo. 
En el primero, ya se indicó, las mujeres simbolizan que la cosmovisión indígena está viva en las prácticas reproductivas que desarrollan las mujeres (y que podrían desarrollar todos los miembros de la comunidad).

En el segundo, y en la medida que las mujeres indígenas son consideradas como las "reproductoras biológicas, culturales y simbólicas" (Yuval-Davis, 2004) de sus pueblos indígenas, su acceso a cargos de representación política extracomunitarios (cualquiera que sea el nivel o ámbito) refleja la imagen (el símbolo) del carácter plurinacional de los Estados.

Asimismo, como destacan Cunningham y Sena (2013), las mujeres indígenas que ejercen sus funciones públicas parecen tener un apego mayor a sus tradiciones culturales (vestimentas, idioma, funciones). Y traigo aquí dos ejemplos paradigmáticos que, entiendo, ayudan a comprender mejor aquella noción de la inscripción simbólica de la plurinacionalidad en la sociedad y la politica.

En primero: Antonia Rodríguez Medrano, Ministra de Desarrollo Productivo y Economía Plural del Estado Plurinacional de Bolivia entre el 2010 y el 2011. Pese a no ser legisladora (tenía funciones en el Poder Ejecutivo), fue la primera persona indígena en presentar un informe de gestión en el Parlamento Nacional en su idioma originario. Ella no definió esta acción como una estrategia política y deliberada de afirmación en el Estado Plurinacional, sino más bien como una estrategia personal para dar veracidad de su informe; lo cierto es que fue un acto de representación simbólica instituyente.

... los diputados me pidieron el informe del Ministerio y allá no había que mentir, no había que maquillar, no había que arreglarlo, adornarlo, entonces yo dije bueno, "voy a hablar en mi idioma" porque me voy a sentir que verdaderamente que voy a hacer y que me pidió... me pidió un diputado que fue de Santa Cruz que no entendía "jota" de quechua, entonces que él para no quedara así, tuvimos que contratar un traductor ¿no? pero yo quería decir, en el informe, la realidad que se vivía, la realidad que se estaba haciendo, y la realidad que yo sentía con el pueblo que estábamos avanzando, entonces elevé mi informe en quechua... (Ciudad de El Alto, Bolivia, octubre del 2011, entrevista realizada por la autora)

El segundo, más reciente: a fines del año 2018 la diputada mapuche Emilia Nuyado, en Chile, se dirigió al ministro del Interior y Seguridad (Andrés Chadwick) en mapudungún, tal como lo había anunciado y pese a las reacciones discriminatorias que esto había generado en una parte significativa de la sociedad chilena, en la sesión de interpelación al funcionario por el asesinato de un comunero mapuche (Camilo Catrillanca).

Ahora bien, la importancia de la representación simbólica de las mujeres indígenas ha sido rápidamente entendida y capturada por los Estados, en general para dilatar las respuestas efectivas a las demandas indígenas, a partir de una incorporación que realza la dimensión simbólica de la representación
Mujeres indígenas y representación política: la dimensión simbólica de su presencia en el espacio público

Andrea Ivanna Gigena 
Dossier América Latina: género y política (particularmente si la encarnan las mujeres), pero sin el reconocimiento de la agencia indígena o de los pueblos indígenas como contraparte.

Esto es la folklorización. El tema ha sido analizado con claridad crítica por Silvia Rivera Cusicanqui en el marco del reconocimiento en clave "multicultural" que las élites estatales latinoamericanas hicieron de las diferencias sociales y culturales en la década de los 90. La autora sostiene que esta perspectiva llevó a considerar a los indígenas como minorías y canalizar su integración mediante políticas "ornamentales y simbólicas" que "teatralizaban la condición originaria" (Rivera Cusicanqui, 2014). Los-as indígenas también advierten esto:

Entonces, a lo mejor si yo me dejara eso, probablemente yo habría estado trabajando en alguna [secretaría] aquí en la región, o me habrían tenido en algún ministerio de agregada cultural a lo mejor, o de asesora, estaría ahí y quizás a lo mejor, me sacaran para todos estos eventos políticos, eventos sociales que hoy día tiene el gobierno, y llegar ahí con toda mi vestimenta al lado de todos los grandes que tienen el poder, para la foto nomás y SIN OPINAR. (Ciudad de Temuco, Chile, junio de 2016, entrevista realizada por la autora)

\section{Conclusión}

La participación política de las mujeres es, claramente, una disputa frente al proceso de asimilación al orden social como masa no protagónica, como la "masa de las representadas" (Ungo, 2000). Y hay varias perspectivas para considerar las formas de representación que se consiguen en esa disputa: que las mujeres han sido electas para un cargo (representación sustantiva) y que las demandas de género se hayan efectivizado (representación sustantiva).

Pero, al considerar a las mujeres indígenas, estas interpretaciones exigen otras consideraciones. La disputa de las mujeres indígenas no es sólo por dejar de ser la "masa de las representadas". También es por dejar de ser "la masa de las representadas" "desmarcadas" de su condición étnica. Por esto he considerado aquella dimensión (o "perspectiva") de la representación, reconocida oportunamente en el tratado de Hanna Pitkin (1985[1967]), pero escasamente analizada luego: la simbólica.

La finalidad ha sido mostrar el variado repertorio de expresiones que la representación asume -de base y territorial, comunitaria, institucional, nacional, internacional- en la práctica regular de las mujeres indígenas por mantener actualizadas las prácticas de sus pueblos, en una lucha por la supervivencia. También, poner en evidencia que las disputas que las mujeres indígenas dan para alcanzar espacios de representación son múltiples: en y frente a sus comunidades o pueblos, frente al Estado, frente a la sociedad nacional e internacional. Y aunque aquí no he reparado en el tema, también frente otras mujeres no indígenas. 
Nada de lo presentado en este artículo debe hacernos suponer que, frente a la simbólica, las otras representaciones no son significativas. Seguramente (aunque algunos-as indígenas que entrevisté manifestaron que las mujeres de sus pueblos no deben participar en instancias de representación extracomunitarias, por ser "débiles", o porque sólo se les atribuyen roles de reproductoras biológicas y culturales) es importante que se generen mecanismos para garantizar un mayor acceso de mujeres indígenas a cargo de elección popular y decisión política. Asimismo, que en el ejercicio de esos cargos tengan las posibilidades efectivas de representar los intereses y las demandas colectivas de sus pueblos (las mixtas), pero también las que les atañen en su singularísima condición de mujeres indígenas.

Y, por supuesto, ejercer siempre una "vigilancia" sobre las prácticas políticas para que las bondades y las potenciales transformadoras de la (auto)representación no sean capturadas por las dinámicas sociales y estatales que pretenden mantener el estatus quo en relación con el avance del reconocimiento de los derechos de los-as indígenas.

\section{Referencias bibliográficas}

Aldunate, V. y Paredes, J. (2010). Construyendo Movimientos. La Paz: Solidaridad Internacional.

Archenti, N. (2002). Los caminos de la inclusión política. Acciones afirmativas de género. En S. Vásquez (Comp.), Hombres públicos, mujeres públicas. Buenos Aires: Fundación Sergio Karakachoff.

CEPAL (2013). Mujeres indígenas en América Latina: dinámicas demográficas y sociales en el marco de los derechos humanos. Recuperado de https://repositorio.cepal.org/bitstream/ handle/11362/4100/1/S2013792_es.pdf

CEPAL (2014). Los pueblos indígenas en América (Abya Yala). Desafíos para la igualdad en la diversidad. Recuperado de https://repositorio.cepal.org/bitstream/handle/11362/43187/6/ S1600364_es.pdf

Cunningham, M. y Sena, K. (2013). Estudio sobre la participación política de las mujeres indígenas a los niveles internacional, nacional y local. Nueva York: Consejo Económico y Social de Naciones Unidas.

Freidenberg, F. y Alva Huitrón, R. (2017). ¡Las reglas importan! Impulsando la representación política de las mujeres desde las leyes electorales en perspectiva multinivel. En F. Freidenberg (Ed.), La representación política de las mujeres en México. Ciudad de México: Instituto Nacional Electoral y Universidad Nacional Autónoma de México.

Fuentes, C. y Sánchez, M. (2018). Asientos reservados para pueblos indígenas. Experiencia comparada, Serie Policy Papers, no. 1, pp. 1-23.

Gigena, A. y de Cea, M. (2018). Institucionalidad indígena / de género y políticas públicas dirigidas a mujeres indígenas en Chile: los casos de CONADI y SERNAM, Revista Perspectivas de Políticas Públicas, vol. 7, no. 14, pp. 323-346.
Mujeres indígenas y representación política: la dimensión simbólica de su presencia en el espacio público

Andrea Ivanna Gigena 
Dossier América Latina: género y política
Gigena, A. (2017). Los-as sujetos-as y los temas ausentes en la Ciencia Política contemporánea. Trabajo presentado para el XIII Congreso Nacional de Ciencia Política, Sociedad Argentina de Análisis Político y la Universidad Torcuato Di Tella, Buenos Aires.

Gigena, A. (2018). Mujeres-Indígenas y decisiones políticas. Alcances y limitaciones de la institucionalización indígena y de género en Chile. Revista de Estudios de Género. La ventana, no. 48 , pp. $390-422$.

Krook, M. (2008). La adopción e impacto de las leyes de cuotas de género: una perspectiva global. En M. Ríos Tobar (Ed.), Mujer y política. El impacto de las cuotas de género en América Latina. Santiago de Chile: FLACSO.

Mairena, E. (2002). Empoderamiento: el empoderamiento para garantizar la plena, activa $y$ propositiva participación de las mujeres indígenas y el fortalecimiento del liderazgo. Trabajo presentado para la Primera Cumbre de Mujeres Indígenas de las Américas, Managua.

ONU MUJERES (2016). Documento Técnico: Los Mecanismos Nacionales y Regionales de las Mujeres en América Latina y el Caribe Hispano. Recuperado de http://www2.unwomen. org/-/media/field\%20office\%20americas/imagenes/publicaciones/2017/final_estudio_ mnm.pdf?la $=$ es\&vs $=0$

Pitkin, H. (1985). El concepto de representación política. Madrid: Centro de Estudios Constitucionales.

PNUD (2014). Representación Indígena en Poderes Legislativos. Claves desde la experiencia internacional.Recuperadodehttp://www.cl.undp.org/content/chile/es/home/library/democratic_governance/serie-mas-y-mejor-democracia/representacion-indigena-en-podereslegislativos.html

Rivera Cusicanqui, S. (2014). Ch’ixinakax utxiwa. Una reflexión sobre prácticas y discursos descolonizados. Buenos Aires: Tinta Limón.

Rodríguez Gustá, A. y Madera, N. (2016). Más allá del recinto legislativo - estrategias colectivas para una agenda de género en América Latina y el Caribe, Revista Sociologías, año 18, no. 17, pp. 356-382. Recuperado de http://dx.doi.org/10.1590/15174522-018004222

Ungo, U. (2000). Para cambiar la vida: política y pensamiento del feminismo en América Latina. Panamá: Instituto de la Mujer de la Universidad de Panamá.

Yuval-Davis, N. (2004). Género y nación. Lima: Flora Tristán. 